\title{
On the Zeros of a Special Sequence of Polynomials
}

\author{
By K. Mahler
}

In memory of my teacher C. L. Siegel

\begin{abstract}
The zeros of the polynomials $P_{n}(z)=\sum_{k=0}^{n-1} z^{2^{k}}$ are studied and a number of open questions are discussed.
\end{abstract}

Let $n$ be a positive integer at least 3 (the cases $n=1$ and $n=2$ are trivial), and let $P_{n}(z)$ be the polynomial

$$
P_{n}(z)=\sum_{k=0}^{n-1} z^{2^{k}}
$$

of degree $2^{n-1}$, so that

$$
P_{n+1}(z)=z+P_{n}\left(z^{2}\right)=P_{n}(z)+z^{2^{n}} .
$$

When $z$ lies in the unit disk $U_{i}:|z|<1$ in the complex plane, the sequence $\left\{P_{n}(z)\right\}$ converges to the power series

$$
f(z)=\sum_{k=0}^{\infty} z^{2^{k}}
$$

which is regular in $U_{i}$, but has the unit circle $U:|z|=1$ as its natural boundary and thus cannot be continued into the exterior $U_{e}:|z|>1$ of this circle (see, e.g., Mahler [5]).

Since $U$ is the circle of convergence of $f(z)$, a general theorem by Jentsch ([3]; see also Landau [4]) implies that the set, $S$ say, of all the zeros of all the polynomials $P_{n}(z)$ is dense in $U$.

Our aim is to discuss the distribution of the elements of $S$ in the complex plane.

1. All the polynomials $P_{n}(z)$ have the trivial zero

$$
z=0 \text {, }
$$

and they further have just one real zero which lies in the open interval $(-1,0)$ and which, as $n$ tends to infinity, tends to the limit

$$
z=-0.6586267543 \ldots,
$$

which is the one real negative zero of $f(z)$. All the other zeros of $P_{n}(z)$ are nonreal and thus form

$$
\frac{2^{n-1}-2}{2}=2^{n-2}-1
$$

Received September 29, 1981.

1980 Mathematics Subject Classification. Primary 65D20. 
pairs of complex conjugate numbers. Denote by $S(n), S_{i}(n)$, and $S_{e}(n)$ the three sets of all these pairs of complex numbers that lie on $U$, in $U_{i}$, and in $U_{e}$, respectively. Let further $N(n), N_{i}(n)$, and $N_{e}(n)$ be the numbers of pairs in $S(n)$, $S_{i}(n)$, and $S_{e}(n)$, respectively; hence

$$
N(n)+N_{i}(n)+N_{e}(n)=2^{n-2}-1
$$

2. The zeros, $\varepsilon$ say, in the set $S(n)$ are of particular interest and lie by the definition of $S(n)$ on the unit circle $U$. These zeros can be divided into two classes $C_{1}$ and $C_{2}$, where the elements of $C_{1}$ are roots of unity, while the elements of $C_{2}$ are not. Here, for the various values of $n$, all three sets $S(n), C_{1}$, and $C_{2}$ may possibly be empty.

A classical theorem by L. Fuchs ([2]; for a modern treatment see Evans [1]) allows us to determine all suffixes $n$ for which the set $C_{1}$ is not empty. Assume that the zero $\varepsilon$ in $C_{1}$ is a primitive $k$ th root of unity. Then

$$
P_{n}(\varepsilon)=\varepsilon+\varepsilon^{2}+\varepsilon^{4}+\cdots+\varepsilon^{2^{n-1}}=0
$$

is a Gaussian cyclotomic period of length $n$. For every odd, positive integer $m$ denote by $t(m)$ the smallest positive integer $t$ such that

$$
2^{t} \equiv 1(\bmod m) \text {. }
$$

By Fuchs's theorem the equation $P_{n}(\varepsilon)=0$ holds if and only if there exists a prime $p$ and a positive, odd integer $k$ such that

$$
p^{2} \mid k \text { and } n=t(k)=p \times t(k / p) .
$$

Denote by $Z_{k}(z)$ the $k$ th cyclotomic polynomial. It is of degree $\phi(k)$, has rational integral coefficients and highest coefficient 1 , has as its roots all the primitive $k$ th roots of unity, and is irreducible over the rational field $Q$.

By way of example, Fuchs's theorem implies that

$$
\begin{array}{ll}
P_{6}(z) & \text { is divisible by } Z_{9}(z), \\
P_{12}(z) & \text { is divisible by } Z_{9}(z) Z_{45}(z), \\
P_{18}(z) & \text { is divisible by } Z_{9}(z) Z_{27}(z) Z_{189}(z), \\
P_{20}(z) & \text { is divisible by } Z_{25}(z) Z_{75}(z), \\
P_{21}(z) & \text { is divisible by } Z_{49}(z)
\end{array}
$$

etc. I am indebted to D. H. Lehmer for a large table of such factors of which these five cases are the first examples.

We see from these factorizations that $P_{6}(z)$ has 3 pairs of complex conjugate cyclotomic roots on $U, P_{12}(z)$ has 15 pairs, $P_{18}(z)$ has 66 pairs, $P_{20}(z)$ has 30 pairs, and $P_{21}(z)$ has 21 pairs of such zeros.

3. There remain the possible zeros in $S(n)$ of class $C_{2}$. The polynomial $z^{-1} P_{n}(z)$ has integral coefficients equal to 1 ; all its zeros are thus algebraic units. Hence the zeros of class $C_{2}$ are algebraic units which are not roots of unity. There are algebraic units of this kind which have the absolute value l; e.g., the number

$$
\left(\rho \cdot 2^{1 / 3}-1\right) /\left(\rho^{2} \cdot 2^{1 / 3}-1\right),
$$


where $\rho$ is a primitive third root of unity, is an algebraic unit of absolute value 1 which is not a root of unity.

It is, however, not obvious whether such a number could in fact be a zero of one of the polynomials $P_{n}(z)$. I therefore raise the following question.

Problem 1. Do there exist suffixes $n$ for which the equation $P_{n}(z)=0$ has a nonreal root of absolute value 1 which is not a root of unity?

4. Consider next the sets $S_{i}(n)$ and $S_{e}(n)$. Denote by $M_{i}(n)$ and $m_{i}(n)$ the maximum and the minimum of $|\varepsilon|$ extended over all the elements $\varepsilon$ of $S_{i}(n)$, and define similarly $M_{e}(n)$ and $m_{e}(n)$ as the maximum and the minimum of $|\varepsilon|$ extended over the elements $\varepsilon$ of $S_{e}(n)$.

Using a programmable calculator (TI 59), I have for $3 \leqslant n \leqslant 8$ determined all the complex zeros of $P_{n}(z)$ and have also obtained selected zeros for larger values of $n$. For $3 \leqslant n \leqslant 8$ the number of pairs of complex conjugate zeros of $P_{n}(z)$ is given in the following little table.

\begin{tabular}{|c|c|c|c|c|c|c|}
\hline$n=$ & 3 & 4 & 5 & 6 & 7 & 8 \\
\hline$N_{i}(n)=$ & 0 & 1 & 2 & 3 & 8 & 13 \\
\hline$N(n)=$ & 0 & 0 & 0 & 3 & 0 & 0 \\
\hline$N_{e}(n)=$ & 1 & 2 & 5 & 9 & 23 & 50 \\
\hline
\end{tabular}

Thus $N_{i}(n)$ is for these values of $n$ smaller than $N_{e}(n)$.

Problem 2. Obtain estimates or asymptotic formulae for $N_{i}(n)$ and $N_{e}(n)$ and decide whether always $N_{i}(n)<N_{e}(n)$.

5. Consider next the minimum $m_{i}(n)$ and the maximum $M_{i}(n)$, using the known zeros of $P_{n}(z)$.

First,

$$
\begin{aligned}
& m_{i}(4)=0.9576366 \ldots \\
& m_{i}(5)=0.9314817 \ldots, \\
& m_{i}(6)=0.9456191 \ldots, \\
& m_{i}(7)=0.9422963 \ldots, \\
& m_{i}(8)=0.9423184 \ldots, \\
& m_{i}(9)=0.9423183 \ldots
\end{aligned}
$$

As $n$ tends to infinity, $m_{i}(n)$ tends to the absolute value

$$
0.942318380 \ldots
$$

of the pair of complex conjugate zeros

$$
0.120314841 \ldots \pm i \cdot 0.934605942 \ldots
$$

of $f(z)$ which is closest to the origin $z=0$. It is interesting to note that the successive values of $m_{i}(n)$ are oscillating. 
Secondly,

$$
\begin{aligned}
M_{i}(4) & =0.9576366 \ldots, \\
M_{i}(5) & =0.9839490 \ldots, \\
M_{i}(6) & =0.9894514 \ldots, \\
M_{i}(7) & =0.9988882 \ldots, \\
M_{i}(8) & =0.9994207 \ldots, \\
M_{i}(9) & =0.9998920 \ldots, \\
M_{i}(10) & =0.9999880 \ldots
\end{aligned}
$$

With increasing $n$ the maximum $M_{i}(n)$ tends rather rapidly to 1 . If the zeros $\varepsilon$ of $P_{n}(z)$ are written in the trigonometric form

$$
\varepsilon=\rho(\cos \phi+i \cdot \sin \phi),
$$

then the arguments $\phi$ at which the maximum $M_{i}(n)$ is attained do not seem to follow any simple law, being roughly $79^{\circ}$ for $n=4,136^{\circ}$ for $n=5,66^{\circ}$ for $n=6,32^{\circ}$ for $n=7,163^{\circ}$ for $n=8,55^{\circ}$ for $n=9,72^{\circ}$ for $n=10$, etc.

6. We come next to the exterior minimum $m_{e}(n)$ and the exterior maximum $M_{e}(n)$. Both tend rather rapidly to 1 as $n$ tends to infinity. For the equation $P_{n}(\varepsilon)=0$ implies that

$$
\varepsilon^{2^{n-2}}=-\left(1+\varepsilon^{2^{n-3}-2^{n-2}}+\varepsilon^{2^{n-4}-2^{n-2}}+\cdots+\varepsilon^{2-2^{n-2}}+\varepsilon^{1-2^{n-2}}\right) .
$$

Here we are dealing with zeros satisfying $|\varepsilon|>1$. Hence the $n-1$ terms on the right-hand side all have at most the absolute value 1 , and it follows that

$$
|\varepsilon| \leqslant(n-1)^{1 / 2^{n-2}} \text {. }
$$

In fact, the convergence to 1 is more rapid. For the maximum $M_{e}(n)$ we obtain the table

$$
\begin{aligned}
& M_{e}(3)=1.2106077 \ldots \\
& M_{e}(4)=1.1560721 \ldots \\
& M_{e}(5)=1.0938325 \ldots \\
& M_{e}(6)=1.0528919 \ldots \\
& M_{e}(7)=1.0289460 \ldots \\
& M_{e}(8)=1.0155673 \ldots \\
& M_{e}(9)=1.0081600 \ldots \\
& M_{e}(10)=1.0043568 \ldots
\end{aligned}
$$

etc. It seems that $M_{e}(n)$ is always attained at that pair of complex conjugate zeros of $P_{n}(z)$ which is closest to the point $z=1$.

The minimum $m_{e}(n)$ tends more rapidly to 1 than $M_{e}(n)$, and again the argument $\phi$ of the zero $\varepsilon$ at which $m_{e}(n)$ is attained does not seem to satisfy any simple law, being roughly $73^{\circ}$ for $n=3,137^{\circ}$ for $n=4,37^{\circ}$ for $n=5,111^{\circ}$ for $n=6,146^{\circ}$ for $n=7,140^{\circ}$ for $n=8,172^{\circ}$ for $n=9,149^{\circ}$ for $n=10,16^{\circ}$ for $n=11$, etc. 
For $m_{e}(n)$ the following table is obtained.

$$
\begin{aligned}
m_{e}(3) & =1.2106077 \ldots \\
m_{e}(4) & =1.1124390 \ldots \\
m_{e}(5) & =1.0192124 \ldots \\
m_{e}(6) & =1.0114222 \ldots \\
m_{e}(7) & =1.0019597 \ldots \\
m_{e}(8) & =1.0004200 \ldots \\
m_{e}(9) & =1.0001314 \ldots \\
m_{e}(10) & =1.0000380 \ldots \\
m_{e}(11) & =1.0000186 \ldots
\end{aligned}
$$

As far as my calculations go, the only zeros of the polynomials $P_{n}(z)$ on the unit circle are roots of unity. Thus, if Problem 1 has a positive answer, $P_{n}(z)$ will be at least of degree 2048, and the suffix $n$ cannot be less than 12 and most probably is very much larger.

7. So far only the absolute values of the zeros have been considered. It seems that the arguments of the zeros are much more uniformly distributed over the values from $0^{\circ}$ to 360 degrees. Actually, since the complex zeros are symmetric in the real axis, it suffices to study the arguments between $0^{\circ}$ and $180^{\circ}$.

Number the arguments $\phi_{N}$ of the successive zeros in the upper halfplane in order of increasing size,

$$
\phi_{1}<\phi_{2}<\cdots<\phi_{P}, \quad \text { where } P=2^{n-2}-1 .
$$

Linear regression statistics gives then for $\phi_{N}$ the following approximate formulae.

$$
\begin{array}{llll}
n=4: & 31.12187+52.08187 N, & \text { correlation coefficient } & 0.99795, \\
n=5: & 14.14548+24.21492 N, & \text { correlation coefficient } & 0.99876, \\
n=6: & 6.65296+11.69893 N, & \text { correlation coefficient } & 0.99923, \\
n=7: & 3.19718+5.73965 N, & \text { correlation coefficient } & 0.99984, \\
n=8: & 1.55480+2.84169 N, & \text { correlation coefficient } & 0.99995 .
\end{array}
$$

At each step the new linear approximation formula is roughly half that of the preceding one.

8. In my paper (Mahler [5]) I gave already the following three pairs of complex zeros of the function $f(z)$.

$$
\begin{array}{rll}
0.1203148 \pm i \cdot 0.9346059, & \rho=0.9423223, & \phi=82.6645070^{\circ}, \\
0.3918627 \pm i \cdot 0.8982576, & \rho=0.9800118, & \phi=66.4300880^{\circ}, \\
-0.6852062 \pm i \cdot 0.6705341, & \rho=0.9587093, & \phi=135.6200425^{\circ} .
\end{array}
$$

Using zeros of the polynomials $P_{n}(z)$ in $U_{i}$ as a first approximation of zeros of $f(z), \mathrm{I}$ can now add the following five pairs of zeros of $f(z)$; I have little doubt that there are infinitely many of them. 


$\begin{array}{rll}0.7754338 \pm i \cdot 0.6181341, & \rho=0.9916589, & \phi=38.5599697^{\circ}, \\ -0.3692106 \pm i \cdot 0.9216532, & \rho=0.9928551, & \phi=111.8308436^{\circ}, \\ 0.7474599 \pm i \cdot 0.6576873, & \rho=0.9956148, & \phi=41.3444314^{\circ}, \\ 0.0819335 \pm i \cdot 0.9936717, & \rho=0.9970439, & \phi=85.2863247^{\circ}, \\ -0.5542160 \pm i \cdot 0.8270115, & \rho=0.9955418, & \phi=123.8277405^{\circ} .\end{array}$

Mathematics Department

Research School of Physical Sciences

Australian National University

Canberra, ACT 2600, Australia

1. R. J. Evans, “Generalized cyclotomic periods," Proc. Amer. Math. Soc., v. 81, 1981, pp. 207-212.

2. I. L. FuCHS, “Über die Perioden, welche aus den Wurzeln der Gleichung $\omega^{n}=1$ gebildet sind," $J$. Reine Angew. Math., v. 61, 1863, 374-386.

3. R. JENTSCH, Untersuchungen zur Theorie der Folgen analytischer Funktionen, Inauguraldissertation Berlin, 1914, $39 \mathrm{~s}$.

4. E. LANDAU, Darstellung und Begründung einiger neuen Ergebnisse der Funktionentheorie, SpringerVerlag, Berlin, 1916.

5. K. MAHLER, “On a special function,” J. Number Theory, v. 12, 1980, pp. 20-26. 